\section{The efficiency of antianemic therapy by Maltofer in the prevention of obstetric and perinatal complications}

Iron deficiency occurs in $20-30 \%$ of all women, $40-50 \%$ in fertile age, and $45-99 \%$ in pregnant women.

The study analyzed the course and outcome of the current pregnancy of 60 patients with anemia, depending on the start of treatment: 35 , enrolled in 6-8 weeks. (I group) and 25 - in 20-25 weeks. Pregnancy (group II).

Conclusion: Thus, the implementation of WHO recommendations on the prevention and treatment of anemia with the onset of the first trimester of pregnancy should be considered a reserve. Medication correction of interruption fetoplacental homeostasis in combination with anti-anemic treatment by Maltofer from early pregnancy has reduced the frequency of obstetric and perinatal complications. A convincing criterion for the effectiveness of the proposed measures was a decrease in the neonatal morbidity by a factor of 1.5 .

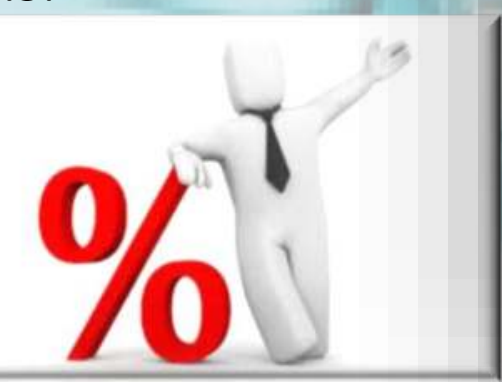

\section{risk of abortion}

$28,60 \%$
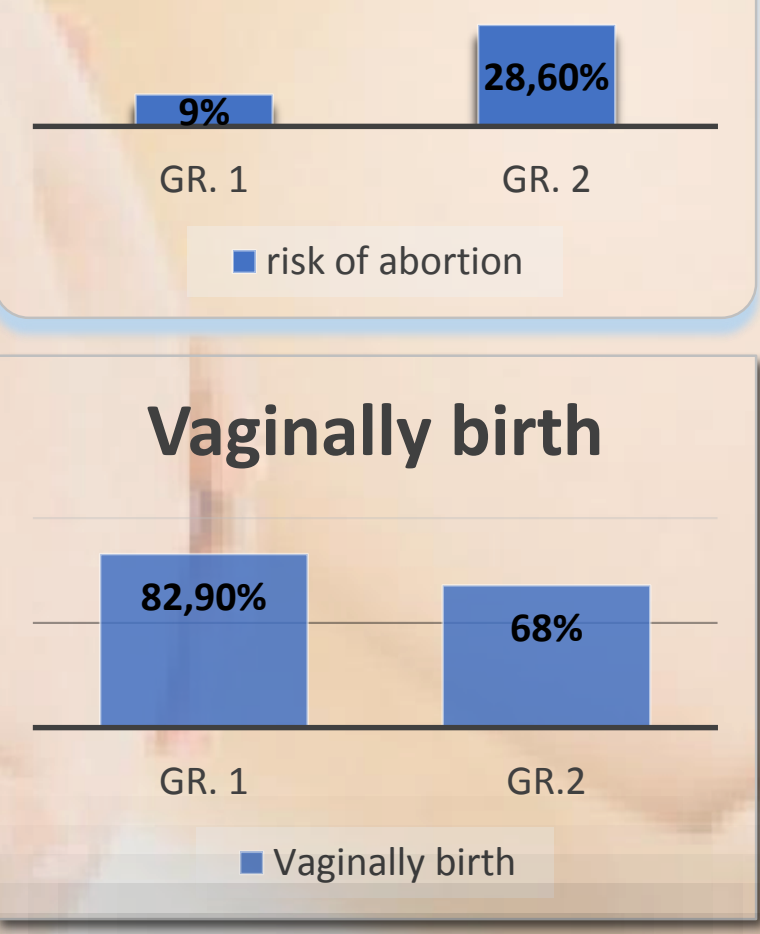

\section{Apgar score}

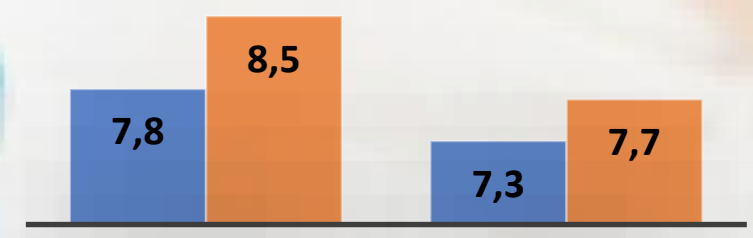

GR.1

GR.2

\section{preeclampsia}

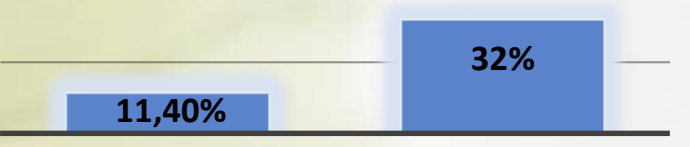

GR. 1

GR.2

- preeclampsia

premature discharge

of amniotic fluid

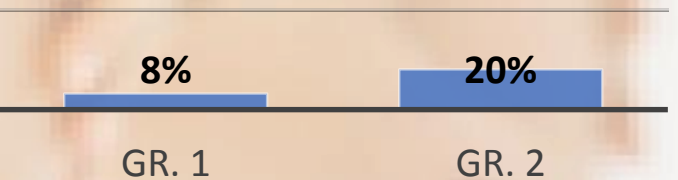

GR. 1

GR. 2

premature discharge of amniotic fluid

newborn asphyxia

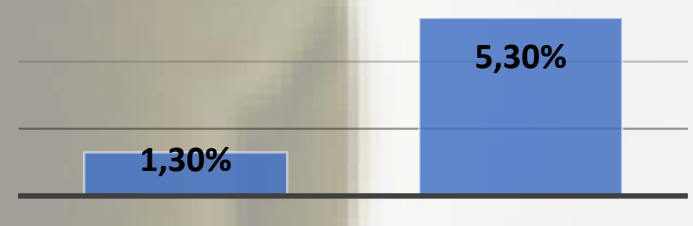

GR. 1

GR. 2 